\title{
Compactness of the Commutators of Fractional Hardy Operator with Rough Kernel
}

\author{
Shaoguang Shi* and Zunwei Fu \\ School of Mathematics and Statistics, Linyi University, Linyi, Shandong 276005, \\ China
}

Received 20 August 2020; Accepted (in revised version) 21 July 2021

Dedicated to Prof. Shanzhen Lu with admiration on the occasion of his 80th birthday

\begin{abstract}
The more explicit decomposition of the operator and the kernel are utilized to investigate a characterization of the central $B M O\left(\mathbb{R}^{n}\right)$-closure of $C_{c}^{\infty}\left(\mathbb{R}^{n}\right)$ space via the compactness of the commutators of fractional Hardy operator with rough kernel.
\end{abstract}

Key Words: Fractional Hardy operator, commutator, compactness.

AMS Subject Classifications: 47B47, 47G10, 42B25

\section{Introduction}

Problem of commutators draws recently more and more attention of Harmonic analysis, such as its application in the study of elliptic equations [1,7]. For example, Sun, Wang and Zhang simplify the proof of the famous Wu's theorem on Navier-Stokes equations greatly in [18] and the technique used is some estimates for commutators by $\mathrm{Lu}$ and Yan [13]. The commutator formed by an operator $T$ and a suitable function $b$ can be recalled as

$$
[b, T] f:=b(T f)-T(b f) .
$$

We call a function $b \in L_{l o c}\left(\mathbb{R}^{n}\right)$ is a central $B M O\left(\mathbb{R}^{n}\right)$ (the mean oscillation function space) function, denoted by $C B M O\left(\mathbb{R}^{n}\right)$ which was introduced by Lu and Yang [14], if

$$
\|b\|_{C B M O\left(\mathbb{R}^{n}\right)}:=\sup _{r>0} \frac{1}{\left|B_{r}\right|} \int_{B_{r}}\left|b(x)-b_{B_{r}}\right| d x<\infty .
$$

Here and in what follows, $B_{r}:=B(0, r)$ is a ball centered at 0 with radius $r>0$. $C B M O\left(\mathbb{R}^{n}\right)$ can be understood as a local version of $B M O\left(\mathbb{R}^{n}\right)$ at the origin, $B M O\left(\mathbb{R}^{n}\right) \subset$ $C B M O\left(\mathbb{R}^{n}\right)$ and they have quite different properties since for $1<p<\infty$,

$$
\|b\|_{B M O\left(\mathbb{R}^{n}\right)} \approx\|b\|_{B M O^{p}\left(\mathbb{R}^{n}\right)} \text { and }\|b\|_{C B M O\left(\mathbb{R}^{n}\right)} \lesssim\|b\|_{C B M O^{p}\left(\mathbb{R}^{n}\right)}
$$

*Corresponding author. Email address: shishaoguang@mail.bnu.edu.cn (S. Shi), fuzunwei@lyu.edu.cn (Z. Fu) 
with

$$
\begin{aligned}
& \|b\|_{B M O^{p}\left(\mathbb{R}^{n}\right)}=\sup _{B \subset \mathbb{R}^{n}}\left(\frac{1}{|B|} \int_{B}\left|b(x)-b_{B}\right|^{p} d x\right)^{\frac{1}{p}}, \\
& \|b\|_{C B M O^{p}\left(\mathbb{R}^{n}\right)}=\sup _{r>0}\left(\frac{1}{\left|B_{r}\right|} \int_{B_{r}}\left|b(x)-b_{B_{r}}\right|^{p} d x\right)^{\frac{1}{p}} .
\end{aligned}
$$

Thus, the John-Nirenberg inequality is not true for $C B M O\left(\mathbb{R}^{n}\right)$. We follow the notation used in the existed work: $V M O\left(\mathbb{R}^{n}\right)$ denotes the $B M O\left(\mathbb{R}^{n}\right)$-closure of $C_{c}^{\infty}\left(\mathbb{R}^{n}\right)$ (the space of all functions being infinite-times continuously differential in $\mathbb{R}^{n}$ with compact support), $C V M O\left(\mathbb{R}^{n}\right)$ stands for the $C B M O\left(\mathbb{R}^{n}\right)$-closure of $C_{c}^{\infty}\left(\mathbb{R}^{n}\right)$.

This paper provides a characterization of the $C V M O\left(\mathbb{R}^{n}\right)$ space by the compactness of $[b, T]$, when $T$ is the following fractional Hardy operator

$$
\begin{aligned}
& H_{\Omega, \alpha} f(x)=\frac{1}{|x|^{n-\alpha}} \int_{|y|<|x|} \Omega(x-y) f(y) d y, \\
& H_{\Omega, \alpha}^{*} f(x)=\int_{|y| \geq|x|} \frac{\Omega(x-y) f(y)}{|y|^{n-\alpha}} d y, \quad 0<\alpha<n .
\end{aligned}
$$

Here $\Omega$ satisfies

$$
\begin{array}{ll}
\Omega(t x)=\Omega(x), & \forall t>0, \quad x \in \mathbb{R}^{n}, \\
\int_{\mathbb{S}^{n-1}} \Omega\left(x^{\prime}\right) d \sigma\left(x^{\prime}\right)=0, & \\
\Omega \in L^{q}\left(\mathbb{S}^{n-1}\right), & \forall q \geq 1 .
\end{array}
$$

The $L^{q \geq 1}$-Dini condition of $\Omega$ can be recalled as

$$
\int_{0}^{1} \frac{w_{q}(\delta)}{\delta}<\infty \quad \text { with } w_{q}(\delta)=\sup _{\|\tau\| \leq \delta}\left(\int_{S^{n-1}}\left|\Omega\left(\tau x^{\prime}\right)-\Omega\left(x^{\prime}\right)\right|^{q} d \sigma\left(x^{\prime}\right)\right)^{\frac{1}{q}}
$$

and $\tau$ is a rotation on $\mathrm{S}^{n-1}$ with

$$
\|\tau\|=\sup _{x^{\prime} \in \mathbb{S}^{n-1}}\left|\tau x^{\prime}-x^{\prime}\right| .
$$

For a suitable function $h, H_{\Omega, \alpha}^{*}$ is said to be the dual operator of $H_{\Omega, \alpha}$ in the following sense

$$
\int_{\mathbb{R}^{n}} h(x) H_{\Omega, \alpha} f(x) d x=\int_{\mathbb{R}^{n}} f(x) H_{\Omega, \alpha}^{*} h(x) d x .
$$

$\mathrm{Fu}, \mathrm{Lu}$ and Zhao considered the boundedness of $H_{\Omega, \alpha}$ and $\left[b, H_{\Omega, \alpha}\right]$ on homogeneous Herz spaces and Lebesgue spaces for $b \in B M O\left(\mathbb{R}^{n}\right)$ in [11]. For $\Omega=1$, see for example $[9,16]$. 
The pioneer work on the compactness of operators can be traced to Uchiyama [19], where a characterization of $V M O\left(\mathbb{R}^{n}\right)$ via the compactness of $[b, T]$ with $T$ is the classical Calderón-Zygmund singular integral operator is obtained. To date, much work has been reported in these field. For example, the compactness of $[b, T]$ on Lebesgue space when $b$ is in an appropriately $B M O$ space and $T$ is the multiplication operator [2]; a characterization of $\operatorname{VMO}\left(\mathbb{R}^{n}\right)$ by the compactness of $[b, T]$ when $T$ is the parabolic singular integral [4]; the compactness theory of $[b, T]$ when $T$ is the generalized Toeplitz operators by Krantzl and Li [12]; the characterizations of $V M O\left(\mathbb{R}^{n}\right)$ via the compactness of $[b, T]$ when $T$ is the Riesz potential [5] and $T$ is the singular integral operator [6] on Morrey type space; the compactness of $[b, T]$ for bilinear operators on Morrey spaces [8]; the characterization of $C V M O\left(\mathbb{R}^{n}\right)$ by compactness of $[b, T]$ when $T$ is the classical Hardy operator and the Hardy operator with homogeneous kernels [10,15].

The know results for the function characterizations highly depended on the smoothness of $\Omega$ and there have been many attempts to weak the condition of $\Omega$ have been undertaken, see e.g., [19] for $\Omega \in \operatorname{Lip}_{1}\left(\mathrm{~S}^{n-1}\right)$ (Lipschitz functional space), [3, 4] for $\Omega$ satisfies

$$
\left|\Omega\left(x^{\prime}\right)-\Omega\left(y^{\prime}\right)\right| \leq \frac{A}{\left(\log \frac{2}{\left|x^{\prime}-y^{\prime}\right|}\right)^{\gamma}} \quad \text { with } A>0, \quad \gamma>1 \quad \text { and } \quad x^{\prime}, y^{\prime} \in \mathbb{S}^{n-1} \text {. }
$$

It is obvious that (1.2) is weaker than the Lipschitz condition $\operatorname{Lip}_{0<\gamma \leq 1}\left(\mathbb{S}^{n-1}\right)$ and is stronger than (1.1c). Furthermore, if $\Omega$ satisfies (1.2), then for $q \geq 1$,

$$
\int_{0}^{1} \frac{w_{q}(\delta)}{\delta}(1+|\log \delta|) d \delta<\infty
$$

The major goal of this paper is to give the following characterization of $C V M O\left(\mathbb{R}^{n}\right)$ via the compactness of $\left[b, H_{\Omega, \alpha}\right]$ and $\left[b, H_{\Omega, \alpha}^{*}\right]$.

Theorem 1.1. Let $0<\alpha<n, \frac{1}{q}=\frac{1}{p}-\frac{\alpha}{n}, \Omega$ satisfy (1.1a), (1.1b), (1.2) and $b \in B M O\left(\mathbb{R}^{n}\right)$. Then $b \in C V M O\left(\mathbb{R}^{n}\right) \Longleftrightarrow$ Both $\left[b, H_{\Omega, \alpha}\right]$ and $\left[b, H_{\Omega, \alpha}^{*}\right]$ are compact from $L^{p}\left(\mathbb{R}^{n}\right)$ to $L^{q}\left(\mathbb{R}^{n}\right)$.

Remark 1.1. The assumption $b \in B M O\left(\mathbb{R}^{n}\right)$ in Theorem 1.1 can not be weakened in the proof of the necessity part since the John-Nirenberg inequality of $B M O\left(\mathbb{R}^{n}\right)$ function is used and it is not true for $C B M O\left(\mathbb{R}^{n}\right)$. Since $H_{\Omega, \alpha}$ is centrosymmetric, the method used to consider the Calderón-Zygmund singular integral [4] can not be applied to $H_{\Omega, \alpha}$ directly.

Section 2 devoted to the basic lemmas for the proof Theorem 1.1; in Section 3, we shall give the proof of Theorem 1.1 by more general case.

In what follows, the symbol $C$ stands for a positive constant which may vary from line to line. $A \lesssim B$ means $A \leq C B$ and $A \simeq B$ whenever $A \lesssim B$ and $B \lesssim A$. $\mathbb{Z}$ denotes the set of all integers. $B_{k}:=B_{2^{k}}, C_{k}:=B_{k} \backslash B_{k-1}$ and $\chi_{k}:=\chi_{C_{k}}$ with $k \in \mathbb{Z}$. 


\section{Preparation}

Four lemmas will be described in this section which are useful for the analysis of Theorem 1.1. We first recall the John-Nirenberg type inequality of $B M O\left(\mathbb{R}^{n}\right)$ function and some properties of $C B M O\left(\mathbb{R}^{n}\right)$ type function from [15, Lemma 2.1].

Lemma 2.1. (a) Let $b \in B M O\left(\mathbb{R}^{n}\right)$. Then for $C_{2}>C_{1}>2$ and $\forall x_{0} \in \mathbb{R}^{n}$, there exist positive constants $C_{3}, C_{4}, C_{5}$ (depending on $C_{1}, C_{2}$ and $b$ ), such that

$$
\begin{aligned}
& \left|\left\{C_{1} r<\left|x-x_{0}\right|\left\langle C_{2} r:\left|b(x)-b_{B\left(x_{0}, r\right)}\right|\right\rangle v+C_{3}\right\}\right| \\
\leq & C_{4}\left|B\left(x_{0}, r\right)\right| e^{-C_{5} v} \text { with } 0<v<\infty .
\end{aligned}
$$

(b) Write

$$
\Phi\left(b, B_{r}\right):=\inf _{c \in \mathbb{R}} \frac{1}{\left|B_{r}\right|} \int_{B_{r}}|b(y)-c| d y
$$

and assume that $b \in C B M O\left(\mathbb{R}^{n}\right)$, then $b \in C V M O\left(\mathbb{R}^{n}\right)$ if and only if $b$ satisfies the following two conditions:

$$
\begin{aligned}
& \limsup _{r \rightarrow 0} \operatorname{su}_{r} \Phi\left(b, B_{r}\right)=0, \\
& \lim _{r \rightarrow \infty} \sup _{r} \Phi\left(b, B_{r}\right)=0 .
\end{aligned}
$$

(c) $\|b\|_{C B M O\left(\mathbb{R}^{n}\right)} \simeq \sup _{r} \Phi\left(b, B_{r}\right)$.

Some estimates for $\Omega$ will be concluded in the next lemma, part of which can be deduced from [10, Lemma 2.1] directly.

Lemma 2.2. Let $\Omega$ satisfy (1.1a) and (1.2). Then

(a) $|\Omega(x-y)-\Omega(x)| \leq \frac{C}{(\log (|x| /|y|))^{\gamma}}$ with $|x| \geq 4|y|$ and $\gamma$ be given in (1.2).

(b) if furthermore $\Omega$ satisfies the $L^{q \geq 1}$-Dini condition, then there is a constant $C>0$ such that for $0<C<1 / 2, r>0, x \in \mathbb{R}^{n}$ with $|x|<C r$, one has

$$
\left\{\begin{array}{l}
\left(\int_{r<|y|<2 r}|\Omega(y-x)-\Omega(y)|^{q} d y\right)^{1 / q} \leq C r^{\frac{n}{q}} \int_{|x| / 2 r}^{|x| / r} \frac{w_{q}(\delta)}{\delta} d \delta \\
\left(\int_{r<|y|<2 r} \frac{|\Omega(y-x)-\Omega(y)|^{q}}{|y|^{(n-\alpha) q}} d y\right)^{1 / q} \leq C r^{-\frac{n}{q^{\prime}}+\alpha} \int_{|x| / 2 r}^{|x| / r} \frac{w_{q}(\delta)}{\delta} d \delta .
\end{array}\right.
$$

Proof. We only need to show the second part of $(b)$ since $(a)$ and the first part of $(b)$ is just [10, Lemma 2.1]. This can be done by the fact that $\Omega$ satisfies the $L^{q}$-Dini condition. 
In fact,

$$
\begin{aligned}
& \left(\int_{r<|y|<2 r} \frac{|\Omega(y-x)-\Omega(y)|^{q}}{|y|^{(n-\alpha) q}} d y\right)^{1 / q} \\
= & C r^{-\frac{n}{q^{\prime}}+\alpha}\left(\int_{r}^{2 r} \int_{\mathbb{S}^{n-1}}\left|\Omega\left(y^{\prime}-t^{-1} x^{\prime}\right)-\Omega\left(y^{\prime}\right)\right|^{q} d \delta\left(y^{\prime}\right) \frac{d t}{t}\right)^{1 / q} \\
\leq & C r^{-\frac{n}{q^{\prime}}+\alpha} \int_{|x| / 2 r}^{|x| / r} \frac{w_{q}(\delta)}{\delta} d \delta .
\end{aligned}
$$

Thus, we complete the proof.

The following known estimates from [17] and [20] will help us to complete the proof of Theorem 1.1.

Lemma 2.3. (a) Let $g(x)$ be a measurable function,

$$
\lambda(\mu)=\left|\left\{x \in \mathbb{R}^{n}:|g(x)|>\mu>0\right\}\right|
$$

and $S$ be a measurable set. Define

$$
g^{*}(t)=\inf \{\mu: \lambda(\mu) \leq t\} \quad \text { for } t>0,
$$

then

$$
\int_{S}|g(x)|^{p} d x \leq \int_{0}^{|S|}\left|g^{*}(t)\right|^{p} d t \quad \text { with } 1 \leq p<\infty .
$$

(b) Let $0<\alpha<n, \frac{1}{q}=\frac{1}{p}-\frac{\alpha}{n}$ and $\Omega$ satisfy (1.1a) and (1.1c). Then both $H_{\Omega, \alpha}$ and $H_{\Omega, \alpha}^{*}$ are bounded operators from $L^{p}\left(\mathbb{R}^{n}\right)$ to $L^{q}\left(\mathbb{R}^{n}\right)$.

In the end of this section, we give the boundedness for the truncated operators of $H_{\Omega, \alpha}$ and $H_{\Omega, \alpha}^{*}$, which can be seen as a fractional case of [10, Lemma 2.5].

Lemma 2.4. Suppose that $0<\alpha<n, \frac{1}{q}=\frac{1}{p}-\frac{\alpha}{n}$ and set

$$
\begin{cases}H_{\Omega, \alpha}^{\eta} f(x)=\frac{1}{|x|^{n-\alpha}} \int_{S_{1}} \Omega(x-z) f(z) d z & \text { with } S_{1}=\{z:|z|<|x|,|x-z|>\eta\}, \\ H_{\Omega, \alpha}^{*, \eta} f(x)=\int_{S_{2}} \frac{\Omega(x-z) f(z)}{|z|^{n-\alpha}} d y & \text { with } S_{2}=\{z:|z| \geq|x|,|x-z|>\eta\} .\end{cases}
$$

If $\Omega$ satisfies (1.1a) and the $L^{q}$-Dini condition, then $H_{\Omega, \alpha}^{\eta}$ and $H_{\Omega, \alpha}^{*, \eta}$ are bounded operators from $L^{p}\left(\mathbb{R}^{n}\right)$ to $L^{q}\left(\mathbb{R}^{n}\right)$.

Proof. We only give the outline of the proof since the similarity, more details see [10, Lemma 2.5]. It is sufficient to show that for $f \in L^{p}\left(\mathbb{R}^{n}\right)$, there are constants $C>0$ satisfying

$$
\left\|H_{\Omega, \alpha}^{\eta} f\right\|_{L^{q}\left(\mathbb{R}^{n}\right)} \leq C\|f\|_{L^{p}\left(\mathbb{R}^{n}\right)} \quad \text { and } \quad\left\|H_{\Omega, \alpha}^{*, \eta} f\right\|_{L^{q}\left(\mathbb{R}^{n}\right)} \leq C\|f\|_{L^{p}\left(\mathbb{R}^{n}\right)} .
$$


Let us first prove the boundedness of $H_{\Omega, \alpha}^{\eta}$ after the decomposition that

$$
\left|H_{\Omega, \alpha}^{\eta} f(x)\right|=\left|H_{\Omega, \alpha} f(y)-H_{\Omega, \alpha} f_{1}(y)-H_{\Omega, \alpha} f_{2}(y)+H_{\Omega, \alpha}^{\eta} f(x)\right|,
$$

where $f_{1}=f \chi_{4 B}, f_{2}=f-f_{1}$ and $B=B(x, \eta / 4)$. Therefore,

$$
\begin{aligned}
\left|H_{\Omega, \alpha}^{\eta} f(x)\right| \leq & \frac{1}{|B|} \int_{B}\left|H_{\Omega, \alpha} f(y)\right| d y+\frac{1}{|B|} \int_{B}\left|H_{\Omega, \alpha} f_{1}(y)\right| d y \\
& \quad+\frac{1}{|B|} \int_{B}\left|H_{\Omega, \alpha} f_{2}(y)-H_{\Omega, \alpha}^{\eta} f(x)\right| d y \\
\leq & M\left(H_{\Omega, \alpha} f\right)(x)+\operatorname{If}(x)+\operatorname{II} f(x) .
\end{aligned}
$$

Combining the $L^{p}$-boundedness of the maximal operator $M$, the $\left(L^{p}, L^{q}\right)$-boundeness of the fractional maximal operator $M_{\alpha}$ and the $\left(L^{p}, L^{q}\right)$-boundeness of $H_{\Omega, \alpha}$ [20], we get

$$
\begin{aligned}
& \left\|M\left(H_{\Omega, \alpha} f\right)\right\|_{L^{q}\left(\mathbb{R}^{n}\right)} \leq\left\|H_{\Omega, \alpha} f\right\|_{L^{q}\left(\mathbb{R}^{n}\right)} \leq C\|f\|_{L^{p}\left(\mathbb{R}^{n}\right)}, \\
& \|I f\|_{L^{q}\left(\mathbb{R}^{n}\right)} \leq C\left\|M_{\alpha}\left(|f|^{p}\right)^{\frac{1}{p}}\right\|_{L^{q}\left(\mathbb{R}^{n}\right)} \leq C\|f\|_{L^{p}\left(\mathbb{R}^{n}\right)} \\
& \|I I f\|_{L^{q}\left(\mathbb{R}^{n}\right)} \leq C\|f\|_{L^{p}\left(\mathbb{R}^{n}\right)}
\end{aligned}
$$

as desired.

The task is now to show the boundedness of $H_{\Omega, \alpha}^{*, \eta}$. Analysis similar to that in the proof of $H_{\Omega, \alpha}^{\eta}$ shows that

$$
\begin{aligned}
& \left|H_{\Omega, \alpha}^{*, \eta} f(x)\right| \leq M\left(H_{\Omega, \alpha}^{*} f\right)(x)+J(f)(x)+J J(f)(x), \\
& \left\|M\left(H_{\Omega, \alpha}^{*} f\right)\right\|_{L^{q}\left(\mathbb{R}^{n}\right)} \leq\left\|H_{\Omega, \alpha}^{*} f\right\|_{L^{q}\left(\mathbb{R}^{n}\right)} \leq C\|f\|_{L^{p}\left(\mathbb{R}^{n}\right)}, \\
& \|J f\|_{L^{q}\left(\mathbb{R}^{n}\right)} \leq C\left\|M_{\alpha}\left(|f|^{p}\right)^{\frac{1}{p}}\right\|_{L^{q}\left(\mathbb{R}^{n}\right)} \leq C\|f\|_{L^{p}\left(\mathbb{R}^{n}\right)} .
\end{aligned}
$$

Set $\widetilde{S_{2}}=\{z:|z| \geq y,|x-z|>\eta\}$, we obtain that

$$
|J J f(x)| \leq \frac{C}{\left|B_{\frac{\eta}{4}}\right|} \int_{B_{\frac{\eta}{4}}}\left|\int_{\widetilde{S_{2}}} \frac{|\Omega(x-y-z)-\Omega(x-z)||f(z)|}{|z|^{n-\alpha}} d z\right| d y .
$$

Accordingly, we conclude from the Minkowski inequality, Lemma 2.2 and the fact $\mid x-$ $z|\geq 3| z \mid / 4$ for $|x|=2^{k_{0}-1} \eta,|z|>\eta$ and $|y|<\eta / 4$ that

$$
\|J J f\|_{L^{q}\left(\mathbb{R}^{n}\right)} \leq C\|f\|_{L^{p}\left(\mathbb{R}^{n}\right)},
$$

whence reaching the required estimation. 


\section{Proof of Theorem 1.1}

We begin with the proof of the necessity of Theorem 1.1 which is partly inspired by [10, Theorem 4.1]. If $\left[b, H_{\Omega, \alpha}\right]$ and $\left[b, H_{\Omega, \alpha}^{*}\right]$ are both compact operators from $L^{p}\left(\mathbb{R}^{n}\right)$ to $L^{q}\left(\mathbb{R}^{n}\right)$, then [20, Theorem 1.1] implies that $b \in C B M O\left(\mathbb{R}^{n}\right)$. For simplicity, we assume that $\|b\|_{C B M O\left(\mathbb{R}^{n}\right)}=1$. According to Lemma 2.1 , we only need to prove that $(2.2 \mathrm{a})-(2.2 \mathrm{~b})$ holds for $b$. This consists of two steps. We follow the notation used in [10, Theorem 4.1].

Step 1-proving that $b$ satisfies (2.2a). If not, then there exists a $\tau>0$ and a sequence of balls $\left\{B_{i}\right\}_{i=1}^{\infty}$ with $\lim _{i \rightarrow \infty} r_{i}=0$, such that for any $i, \Phi\left(b, B_{i}\right)>\tau$. Upon writing

$$
\begin{gathered}
f_{i}(y)=\frac{1}{\left|B_{i}\right|^{\frac{1}{p}}}\left[\operatorname{sgn}\left(b(y)-b_{B_{i}}\right)-a_{0}\right] \chi_{B_{i}}(y), \quad i=1,2, \cdots, \\
\text { with } a_{0}=\frac{1}{\left|B_{i}\right|} \int_{B_{i}} \operatorname{sgn}\left(b(y)-b_{B_{i}}\right) d y,
\end{gathered}
$$

we find

$$
\left\{\begin{array}{l}
\operatorname{supp} f_{i} \subset B_{i}, \quad f_{i}(y)\left(b(y)-b_{B_{i}}\right)>0 \\
\left|f_{i}(y)\right| \leq 2\left|B_{i}\right|^{-\frac{1}{p}} \quad \text { with } y \in B_{i} \\
\left\|f_{i}\right\|_{L^{p}\left(\mathbb{R}^{n}\right)} \leq C, \quad \int_{\mathbb{R}^{n}} f_{i}(y) d y=0
\end{array}\right.
$$

The argument is completed by showing that $\left\{\left[b, H_{\Omega, \alpha}\right] f_{i}\right\}_{i=1}^{\infty}$ is not a compact set from $L^{p}\left(\mathbb{R}^{n}\right)$ to $L^{q}\left(\mathbb{R}^{n}\right)$. From now on, $C_{k},(k \in \mathbb{Z})$ stands for a positive constant depending only on $\Omega, p, \alpha, \tau$ with $C_{i},(1 \leq i<k)$. We continue to choose

$$
\left\{\begin{array}{l}
D=\left\{x^{\prime} \in \mathbb{S}^{n-1}: \Omega\left(x^{\prime}\right) \geq \frac{2 A}{\left(\log \left(2 / C_{1}\right)\right)^{\gamma}}\right\} \text { with } A, \gamma \text { be the same as that of in (1.2), } \\
E=\left\{x \in \mathbb{R}^{n}:|x|>C_{2} r, x^{\prime} \in D\right\} \quad \text { with } C_{2}=3 C_{1}^{-1}+1>4 .
\end{array}\right.
$$

Using (1.1b) and (1.2), we obtain that there exists a $0<C_{1}<1$ such that

$$
\sigma(D)>0, \quad|x|>C_{2}|y| \text { for } y \in B_{i}, \quad x \in E .
$$

In view of the fact that

$$
\Omega\left(x^{\prime}\right) \geq \frac{2 A}{\left(\log \left(2 / C_{1}\right)\right)^{\gamma}}
$$

and (1.2), we are interested in finding that for $x^{\prime} \in D$ and $y^{\prime} \in \mathbb{S}^{n-1}$ with $\left|x^{\prime}-y^{\prime}\right| \leq C_{1}$,

$$
\Omega\left(y^{\prime}\right)=\Omega\left(x^{\prime}\right)-\left(\Omega\left(x^{\prime}\right)-\Omega\left(y^{\prime}\right)\right) \geq\left|\Omega\left(x^{\prime}\right)\right|-\left|\Omega\left(x^{\prime}\right)-\Omega\left(y^{\prime}\right)\right| \geq \frac{A}{\left(\log \left(2 / C_{1}\right)\right)^{\gamma}} .
$$

This in turn implies that

$$
\Omega\left((x-y)^{\prime}\right) \geq \frac{A}{\left(\log \left(2 / C_{1}\right)\right)^{\gamma}} .
$$


And hence, we get from (3.1) that for $x \in E$,

$$
H_{\Omega, \alpha}\left(\left(b-b_{B_{i}}\right) f_{i}\right)(x) \geq \frac{C}{\left|B_{i}\right|^{\frac{1}{p}}|x|^{n-\alpha}} \int_{B_{i}}\left(\left|b(y)-b_{B_{i}}\right|-a_{0}\left(b(y)-b_{B_{i}}\right)\right) d y .
$$

Consequently,

$$
H_{\Omega, \alpha}\left(\left(b-b_{B_{i}}\right) f_{i}\right)(x) \geq \frac{C\left|B_{i}\right|^{1 / p^{\prime}}}{|x|^{n-\alpha}} \Phi\left(b, B_{i}\right) \geq \frac{C \tau\left|B_{i}\right|^{1 / p^{\prime}}}{|x|^{n-\alpha}} .
$$

On the other hand, (3.1) and Hölder's inequality allow us to obtain

$$
\begin{aligned}
& \left|H_{\Omega, \alpha}\left(\left(b-b_{B_{i}}\right) f_{i}\right)(x)\right| \\
\leq & \frac{1}{|x|^{n-\alpha}} \int_{B_{i}}\left|\Omega\left((x-y)^{\prime}\right)\left(b(y)-b_{B_{i}}\right) f_{i}(y)\right| d y \\
\leq & \frac{C\left|B_{i}\right|^{1 / p^{\prime}}}{|x|^{n-\alpha}}\left(\frac{1}{\left|B_{i}\right|} \int_{B_{i}}\left|b(y)-b_{B_{i}}\right|^{p^{\prime}} d y\right)^{1 / p^{\prime}}\left(\int_{B_{i}}\left|f_{i}(y)\right|^{p} d y\right)^{1 / p},
\end{aligned}
$$

namely,

$$
\left|H_{\Omega, \alpha}\left(\left(b-b_{B_{i}}\right) f_{i}\right)(x)\right| \leq \frac{C\left|B_{i}\right|^{1 / p^{\prime}}}{|x|^{n-\alpha}} .
$$

At the same time, Lemma 2.2(a) and (3.1) shows

$$
\begin{aligned}
& \left|\left(b(x)-b_{B_{i}}\right) H_{\Omega, \alpha}\left(f_{i}\right)(x)\right| \\
\leq & \frac{C\left|b(x)-b_{B_{i}}\right|}{|x|^{n-\alpha}} \int_{B_{i}} \frac{\left|f_{i}(y)\right|}{\left(\log \left(|x| / r_{i}\right)\right)^{\gamma}} d y \leq \frac{C\left|b(x)-B_{i}\right|\left|B_{i}\right|^{1 / p^{\prime}}}{|x|^{n-\alpha}\left(\log \left(|x| / r_{i}\right)\right)^{\gamma}} .
\end{aligned}
$$

This in turn implies that for $a>C_{2}$,

$$
\left(\int_{\left\{|x|>a r_{i}\right\}}\left|\left(b(x)-b_{B_{i}}\right) H_{\Omega, \alpha}\left(f_{i}\right)(x)\right|^{q} d x\right)^{1 / q} \leq C(\log a)^{1-\gamma} a^{-\frac{n}{p^{\prime}}},
$$

where we used the fact that for $O=\left\{x: 2^{m} r_{i}<|x|<2^{m+1} r_{i}\right\}$,

$$
\int_{O}\left|b(x)-b_{B_{i}}\right|^{q} d x \leq \int_{O}\left|b(x)-b_{2^{m} B_{i}}\right|^{q} d x+\int_{O}\left|b_{2^{m} B_{i}}-b_{B_{i}}\right|^{q} d x \leq C m^{q}\left|2^{m} B_{i}\right| .
$$

Upon setting $W=\left\{x: a r_{i}<|x|<b r_{i}\right\}$, we find according to the above analysis that for $b>a>C_{2}$

$$
\begin{aligned}
& \left(\int_{W}\left|\left[b, H_{\Omega, \alpha}\right] f_{i}(x)\right|^{q} d x\right)^{1 / q} \\
\geq & C \tau\left|B_{i}\right|^{\frac{1}{p^{\prime}}}\left(\int_{W \cap\left\{x: x^{\prime} \in D\right\}} \frac{d x}{|x|^{(n-\alpha) q}}\right)^{1 / q}-C(\log a)^{1-\gamma} a^{-\frac{n}{p^{\prime}}} \\
\geq & C \tau\left(a^{-\frac{n q}{p^{\prime}}}-b^{-\frac{n q}{p^{\prime}}}\right)^{1 / q}-C(\log a)^{1-\gamma} a^{-\frac{n}{p^{\prime}}} .
\end{aligned}
$$


At the same time, (3.2) shows that

$$
\begin{aligned}
& \left(\int_{\left\{|x|>b r_{i}\right\}}\left|\left[b, H_{\Omega, \alpha}\right] f_{i}(x)\right|^{q} d x\right)^{1 / q} \\
\leq & \left(\int_{\left\{|x|>b r_{i}\right\}} \frac{\left|B_{i}\right|^{\frac{q}{p^{\prime}}}}{|x|^{(n-\alpha) q}} d x\right)^{1 / q}+C(\log b)^{1-\gamma} b^{-\frac{n}{p^{\prime}}} \\
\leq & C b^{-\frac{n}{p^{\prime}}}+C(\log b)^{1-\gamma} b^{-\frac{n}{p^{\prime}}} .
\end{aligned}
$$

Accordingly, there are constants $C_{3}>C_{2}, C_{5}$ and $C:=C(\Omega, p, n, \alpha, \tau)>1$ with $C_{4}=C C_{3}$ such that

$$
\begin{aligned}
& \left(\int_{\left\{C_{3} r_{i}<|x|<C_{4} r_{i}\right\}}\left|\left[b, H_{\Omega, \alpha}\right] f_{i}(x)\right|^{q} d x\right)^{1 / q} \geq C_{5}, \\
& \left(\int_{\left\{|x|>C_{4} r_{i}\right\}}\left|\left[b, H_{\Omega, \alpha}\right] f_{i}(x)\right|^{q} d x\right)^{1 / q} \leq \frac{C_{5}}{4} .
\end{aligned}
$$

Set $S \subset\left\{x: C_{3} r_{i}<|x|<C_{4} r_{i}\right\}$ be an arbitrary measurable set. An application of the Minkowski inequality shows that

$$
\left(\int_{S}\left|\left[b, H_{\Omega, \alpha}\right] f_{i}(x)\right|^{q} d x\right)^{1 / q} \leq C\left(\frac{|S|}{\left|B_{i}\right|}\right)^{1 / q}+C\left(\frac{1}{\left|B_{i}\right|} \int_{S}\left|b(x)-b_{B_{i}}\right|^{q} d x\right)^{1 / q} .
$$

\section{Setting}

$$
g_{i}(x)=b(x)-b_{B_{i}} \quad \text { and } \quad \lambda_{g_{i}}(t)=\left|\left\{C_{5} r_{i}<|x|<C_{6} r_{i}:\left|g_{i}(x)\right|>t\right\}\right|, \quad 0<t<\infty \text {, }
$$

we obtain from Lemma 2.1 that there are constants $C_{6}, C_{7}$ and $C_{8}$ such that

$$
\lambda_{g_{i}}\left(t+C_{6}\right) \leq C_{7}\left|B_{i}\right| e^{-C_{8} t} \Rightarrow \lambda_{g_{i}}(t) \leq C_{7}\left|B_{i}\right| e^{-C_{8}\left(t-C_{6}\right)} .
$$

Upon choosing $g_{i}^{*}(\mu)=\inf \left\{t: \lambda_{g_{i}}(t) \leq \mu\right\}$, it is easy to check that for $0<\mu<C_{7}\left|B_{i}\right|$,

$$
g_{i}^{*}(\mu) \leq \frac{1}{C_{8}} \ln \frac{C_{7}\left|B_{i}\right|}{\mu}+C_{6}
$$

Using Lemma 2.3, we get that for $|S| \ll C_{7}\left|B_{i}\right|$,

$$
\begin{aligned}
\frac{1}{\left|B_{i}\right|} \int_{S}\left|b(x)-b_{B_{i}}\right|^{q} d x & \leq \frac{1}{\left|B_{i}\right|} \int_{0}^{|S|}\left|g_{i}^{*}(\mu)\right|^{q} d \mu \\
& \leq \frac{C|S|}{\left|B_{i}\right|}\left|1+\ln \left(C_{7}\left|B_{i}\right| /|S|\right)\right|^{[q]+1} .
\end{aligned}
$$


Eqs. (3.4) and (3.5) imply that there is a $C_{9}<\min \left\{C_{7}^{\frac{1}{n}}, C_{4}\right\}$ such that for $|S| /\left|B_{i}\right|<C_{9}^{n}$,

$$
\begin{aligned}
& \left(\int_{S}\left|\left[b, H_{\Omega, \alpha}\right] f_{i}(x)\right|^{q} d x\right)^{1 / q} \\
\leq & C\left(\frac{|S|}{\left|B_{i}\right|}\right)^{1 / q}+C\left(\frac{|S|}{\left|B_{i}\right|}\left(1+\ln \frac{C_{7}\left|B_{i}\right|}{|S|}\right)^{[q]+1}\right)^{1 / q} \leq \frac{C_{5}}{4} .
\end{aligned}
$$

Picking a subsequence $\left\{B_{i(m)}\right\}_{m}$ from $\left\{B_{i}\right\}$ with $r_{i(m+1)} / r_{i(m)}<C_{9} / C_{4}$, we concluded that for $k>0$,

$$
\begin{aligned}
& \left\|\left[b, H_{\Omega, \alpha}\right] f_{i(m)}-\left[b, H_{\Omega, \alpha}\right] f_{i(m+k)}\right\|_{L^{q}\left(\mathbb{R}^{n}\right)} \\
\geq & \left(\int_{\mathbb{G}_{1}}\left|\left[b, H_{\Omega, \alpha}\right] f_{i(m)}(x)\right|^{q} d x\right)^{1 / q}-\left(\int_{\mathbb{G}_{2}}\left|\left[b, H_{\Omega, \alpha}\right] f_{i(m+k)}(x)\right|^{q} d x\right)^{1 / q},
\end{aligned}
$$

where

$$
\left\{\begin{array}{l}
\mathbb{G}_{1}=\left\{x: C_{5} r_{i(m)}<|x|<C_{6} r_{i(m)}\right\} \backslash\left\{x:|x| \leq C_{6} r_{i(m+k)}\right\}=\mathbb{G}-\left(\mathbb{G}_{2}^{c} \cap \mathbb{G}\right), \\
\mathbb{G}_{2}=\left\{x:|x|>C_{6} r_{i(m+k)}\right\}, \\
\mathbb{G}=\left\{x: C_{5} r_{i(m)}<|x|<C_{6} r_{i(m)}\right\} .
\end{array}\right.
$$

From (3.3) and what already been proved, we conclude that

$$
\left\|\left[b, H_{\Omega, \alpha}\right] f_{i(m)}-\left[b, H_{\Omega, \alpha}\right] f_{i(m+k)}\right\|_{L^{q}\left(\mathbb{R}^{n}\right)} \geq\left(C_{5}^{p}-\left(\frac{C_{5}}{4}\right)^{q}\right)^{1 / q}-\frac{C_{5}}{4} \geq \frac{C_{5}}{4},
$$

which clearly shows that $\left\{\left[b, H_{\Omega, \alpha}\right] f_{i(m)}\right\}_{m=1}^{\infty}$ does not have any convergence subsequence in $L^{q}\left(\mathbb{R}^{n}\right)$. This in turn implies that $\left[b, H_{\Omega, \alpha}\right]$ is not a compact operator from $L^{p}\left(\mathbb{R}^{n}\right)$ to $L^{q}\left(\mathbb{R}^{n}\right)$. Therefore, $b$ satisfies (2.2a) by the contradiction.

Step 2-showing that $b$ satisfies (2.2b). This step can be handled in much the same way as the argument for (2.2a), the only difference being in choosing a sequence $\left\{B_{i}\right\}_{i}$ such that

$$
\Phi\left(b, B_{i}\right)>\tau \text { with } \lim _{i \rightarrow \infty} r_{i}=+\infty .
$$

We proceed to show the sufficiency of Theorem 1.1, which can be deduced by the following more general form.

Theorem 3.1. Suppose that

$$
\left\{\begin{array}{l}
0<\alpha<n, \quad \frac{1}{q}=\frac{1}{p}-\frac{\alpha}{n^{\prime}} \\
\Omega \text { satisfies (1.1a) and (1.3), } \\
b \in \operatorname{CVMO}\left(\mathbb{R}^{n}\right),
\end{array}\right.
$$

then both $\left[b, H_{\Omega, \alpha}\right]$ and $\left[b, H_{\Omega, \alpha}^{*}\right]$ are compact operators from $L^{p}\left(\mathbb{R}^{n}\right)$ to $L^{q}\left(\mathbb{R}^{n}\right)$. 
To prove Theorem 3.1, the following two lemmas are needed. We first recall the well known Frechet-Kolmogorov theorem as

Lemma 3.1. Let a set $S \subset L^{p}\left(\mathbb{R}^{n}\right)$ and $G_{\alpha}=\left\{x \in \mathbb{R}^{n}:|x|>\beta\right\}$. Then $S$ is strongly pre-compact, if and only if,

$$
\begin{aligned}
& \sup _{f \in S}\|f\|_{L^{p}\left(\mathbb{R}^{n}\right)}<\infty, \\
& \lim _{|y| \rightarrow 0}\|f(\cdot+y)-f(\cdot)\|_{L^{p}\left(\mathbb{R}^{n}\right)}=0 \quad \text { uniformly in } f \in S, \\
& \lim _{\beta \rightarrow \infty}\left\|f \chi_{G_{\beta}}\right\|_{L^{p}\left(\mathbb{R}^{n}\right)}=0 \quad \text { uniformly in } f \in S .
\end{aligned}
$$

Next, we give the second lemma which can simplify the proof of Theorem 3.1 by considering $b \in C_{c}^{\infty}\left(\mathbb{R}^{n}\right)$ ([10, Lemma 4.4]).

Lemma 3.2. Assume that $[b, T]$ is a compact operator from $L^{p}\left(\mathbb{R}^{n}\right)$ to $L^{q}\left(\mathbb{R}^{n}\right)$ for $b \in C_{c}^{\infty}\left(\mathbb{R}^{n}\right)$, then $[b, T]$ is also a compact operator from $L^{p}\left(\mathbb{R}^{n}\right)$ to $L^{q}\left(\mathbb{R}^{n}\right)$ for $b \in C V M O\left(\mathbb{R}^{n}\right)$.

We are now in a position to complete the proof of Theorem 3.1. For $b \in C_{c}^{\infty}\left(\mathbb{R}^{n}\right)$, we are about to show (3.6a)-(3.6c) for

$$
\begin{gathered}
S_{1}=\left\{\left[b, H_{\Omega, \alpha}\right] f: f \in Q\right\} \quad \text { and } S_{2}=\left\{\left[b, H_{\Omega, \alpha}^{*}\right] f: f \in Q\right\}, \\
\text { with } Q=\left\{f: f \in L^{p}\left(\mathbb{R}^{n}\right) \text { and }\|f\|_{L^{p}\left(\mathbb{R}^{n}\right)} \leq C\right\}
\end{gathered}
$$

The fact $b \in C_{c}^{\infty}\left(\mathbb{R}^{n}\right)$ allows us to have

$$
\sup _{f \in Q}\left\|\left[b, H_{\Omega, \alpha}\right] f\right\|_{L^{q}\left(\mathbb{R}^{n}\right)} \leq C\|b\|_{C B M O\left(\mathbb{R}^{n}\right)} \sup _{f \in Q}\|f\|_{L^{p}\left(\mathbb{R}^{n}\right)}<\infty
$$

and to obtain (3.6a).

Next, to show (3.6b), we only need to prove that for any $\varepsilon>0$ and $|z|$ small enough,

$$
\left\|\left[b, H_{\Omega, \alpha}\right] f(\cdot+z)-\left[b, H_{\Omega, \alpha}\right] f(\cdot)\right\|_{L^{q}\left(\mathbb{R}^{n}\right)} \leq C \varepsilon, \quad \forall f \in Q .
$$

For $0<\varepsilon<1 / 2$, setting

$$
\begin{cases}E_{1}=\left\{y:|y|<|x+z|,|x-y|>e^{\frac{1}{\varepsilon}}|z|\right\}, & E_{2}=\left\{y:|y|<|x+z|,|x-y| \leq e^{\frac{1}{\varepsilon}}|z|\right\}, \\ E_{3}=\left\{y:|y|<|x|,|x-y|>e^{\frac{1}{\varepsilon}}|z|\right\}, & E_{4}=\left\{y:|y|<|x|,|x-y| \leq e^{\frac{1}{\varepsilon}}|z|\right\},\end{cases}
$$

we achieve that for $z \in \mathbb{R}^{n}$,

$$
\left|\left[b, H_{\Omega, \alpha}\right] f(x+z)-\left[b, H_{\Omega, \alpha}\right] f(x)\right|=K_{1}^{b} f+K_{2}^{b} f+K_{3}^{b} f-K_{4}^{b} f,
$$


where

$$
\left\{\begin{aligned}
K_{1}^{b} f= & \frac{1}{|x|^{n-\alpha}} \int_{E_{3}}[\Omega(x-y)(b(x+z)-b(x))] f(y) d y, \\
K_{2}^{b} f= & \frac{1}{|x|^{n-\alpha}} \int_{E_{3}}[\Omega(x-y)(b(y)-b(x+z))] f(y) d y \\
& -\frac{1}{|x+z|^{n-\alpha}} \int_{E_{1}}[\Omega(x+z-y)(b(y)-b(x+z))] f(y) d y, \\
K_{3}^{b} f= & \frac{1}{|x|^{n-\alpha}} \int_{E_{4}}[\Omega(x-y)(b(y)-b(x))] f(y) d y, \\
K_{4}^{b} f= & \frac{1}{|x+z|^{n-\alpha}} \int_{E_{2}}[\Omega(x+z-y)(b(y)-b(x+z))] f(y) d y .
\end{aligned}\right.
$$

Combining $b \in C_{c}^{\infty}\left(\mathbb{R}^{n}\right),|b(x+z)-b(x)| \leq C|z|$ and Lemma 2.4, we obtain that for $f \in Q$,

$$
\left\|K_{1}^{b} f\right\|_{L^{q}\left(\mathbb{R}^{n}\right)} \leq C|z|\left\|H_{\Omega, \alpha}^{e^{\frac{1}{\varepsilon}}|z|} f\right\|_{L^{q}\left(\mathbb{R}^{n}\right)} \leq C|z|\|f\|_{L^{p}\left(\mathbb{R}^{n}\right)} \leq C|z| .
$$

By Lemma 2.2 and Minkoski's inequality, one has

$$
\begin{aligned}
\left\|K_{2}^{b} f\right\|_{L^{q}\left(\mathbb{R}^{n}\right)} & \leq\left(\int_{x:|x-y|>e^{\frac{1}{\varepsilon}}|z|}\left|\frac{1}{|x|^{n-\alpha}} \int_{\widetilde{U}_{1}}[\Omega(y)-\Omega(y+z)] f(x-y) d y\right|^{q} d x\right)^{1 / q} \\
& \leq C \sum_{k=0}^{\infty} \frac{1}{1+k+\frac{1}{\varepsilon}} \int_{\frac{1}{2^{k+1} e^{\frac{1}{\varepsilon}}}}^{\frac{1}{k^{\frac{1}{\varepsilon}}}} \frac{w_{q}(\delta)}{\delta}(1+|\log \delta|) d \delta \\
& \leq C \varepsilon,
\end{aligned}
$$

where

$$
\widetilde{E_{1}}:=\left\{y: 2^{k+1} e^{\frac{1}{\varepsilon}}|z|<|y|<2^{k} e^{\frac{1}{\varepsilon}}|z|\right\} .
$$

After the observation $|b(x)-b(y)| \leq C|x-y|$ for $|x-y|<1$, we can estimate $K_{3}^{b}$ as

$$
\left|K_{3}^{b} f\right| \leq \frac{C}{|x|^{n-\alpha}} \int_{E_{4}}|\Omega(x-y) f(y)| x-y|| d y \leq \frac{C}{|x|^{n-\alpha-1}} \int_{E_{4}}|\Omega(x-y) f(y)| d y .
$$

Hence, a further application of the Minkoski inequality and the Hölder inequality show that

$$
\begin{aligned}
\left\|K_{3}^{b} f\right\|_{L^{q}\left(\mathbb{R}^{n}\right)} & \leq C\left(\int_{x:|x-y| \leq e^{\frac{1}{\varepsilon}}|z|}\left|\frac{1}{|x|^{n-\alpha-1}} \int_{E_{4}}\right| \Omega(x-y) f(y)|d y|^{q} d x\right)^{1 / q} \\
& \leq C\left(e^{\frac{1}{\varepsilon}}|z|\right)^{q} .
\end{aligned}
$$

Since

$$
|x-y+z| \leq\left(e^{\frac{1}{\varepsilon}}+1\right)|z|<1, \quad|b(x+z)-b(y)| \leq C|x+z-y|,
$$


we can estimate $K_{4}^{b} f$ as follows,

$$
\begin{aligned}
\left\|K_{4}^{b} f\right\|_{L^{q}\left(\mathbb{R}^{n}\right)} & \leq C\left(\int_{\mathbb{R}^{n}}\left|\int_{E_{2}} \frac{|\Omega(x+z-y) f(y)|}{|x+z-y|^{n-\alpha-1}} d y\right|^{q} d x\right)^{1 / q} \\
& \leq C\|f\|_{L^{p}\left(\mathbb{R}^{n}\right)}\left(\int_{E_{2}} d y\right)^{\frac{1}{p^{\prime}}}\left(\int_{\left\{x:|x-y|<e^{\frac{1}{\varepsilon}}|z|\right\}} \frac{|\Omega(x+z-y)|^{q}}{|x+z-y|^{(n-\alpha-1) q}} d x\right)^{\frac{1}{q}} \\
& \leq C\left(\left(e^{\frac{1}{\varepsilon}}+1\right)|z|\right)^{q} .
\end{aligned}
$$

So, (3.7) is obtained thanking to

$$
\lim _{|z| \rightarrow 0}\left\|\left[b, H_{\Omega, \alpha}\right] f(x)-\left[b, H_{\Omega, \alpha}\right] f(x+z)\right\|_{L^{q}\left(\mathbb{R}^{n}\right)}=0 \quad \text { uniformly in } f \in Q .
$$

Next, we finish the consideration of $S_{1}$ by showing (3.6c). To do so, we first choose $\beta$ large enough such that

$$
\left(\int_{\beta}^{\infty} \frac{1}{t^{(n-\alpha) q-n+1}} d t\right)^{\frac{1}{s}}<\varepsilon, \quad \forall \varepsilon>0, \quad s>1,
$$

and denote by $U:=\operatorname{supp}(b) \subset\{x:|x|<r\}$ for some $r>0$. Then for $|x|>\max \{\beta, 4 r\}$ and $f \in Q$, apply the Hölder inequality to $\frac{1}{s}+\frac{1}{p}+\frac{1}{q}=1$, one has

$$
\begin{aligned}
\left|\left[b, H_{\Omega, \alpha}\right] f(x)\right| & \leq \frac{C}{|x|^{n-\alpha}} \int_{U}|b(y) \Omega(x-y) f(y)| d y \\
& \leq \frac{C\|f\|_{L^{p}\left(\mathbb{R}^{n}\right)}}{|x|^{n-\alpha}}\left(\int_{U}|\Omega(x-y)|^{q} d y\right)^{\frac{1}{q}} .
\end{aligned}
$$

Thereby reaching (3.6c) by the Minkoskin inequality and the fact $|x-y|>3|x| / 4$ as

$$
\begin{aligned}
\left(\int_{|x|>\beta}\left|\left[b, H_{\Omega, \alpha}\right] f(x)\right|^{q} d x\right)^{1 / q} & \leq C\left(\left.\int_{|x|>\beta}\left|\frac{1}{|x|^{n-\alpha}} \int_{U}\right| \Omega(x-y)\right|^{q} d y \mid d x\right)^{1 / q} \\
& \leq C\left(\int_{\beta}^{\infty} \frac{d t}{t^{(n-\alpha) q-n+1}} \int_{S^{n-1}}\left|\Omega\left(y^{\prime}\right)\right|^{q} d \sigma\left(y^{\prime}\right)\right)^{\frac{1}{q}} \\
& \leq C \varepsilon .
\end{aligned}
$$

Similar arguments apply to $S_{2}$, we have

$$
\sup _{f \in Q}\left\|\left[b, H_{\Omega, \alpha}^{*}\right] f\right\|_{L^{q}\left(\mathbb{R}^{n}\right)} \leq C \sup _{f \in Q}\|f\|_{L^{p}\left(\mathbb{R}^{n}\right)} \leq C<\infty,
$$

whence finding (3.6a). Since $\{y:|y| \geq|x|\} \cap\{y:|y|<R\}=\phi$ if $U:=\operatorname{supp}(b) \subset\{y$ : $|y|<r\}$ for some $r>0$ and $x$ satisfying $|x|>\max \{\beta, 4 r\}$ in this case, (3.6c) is obviously. 
It is sufficient to prove (3.6b) for $S_{2}$. We are about to show that for any $\varepsilon>0, f \in Q$ and $|z|$ small enough,

$$
\left\|\left[b, H_{\Omega, \alpha}^{*}\right] f(\cdot+z)-\left[b, H_{\Omega, \alpha}^{*}\right] f(\cdot)\right\|_{L^{q}\left(\mathbb{R}^{n}\right)} \leq C \varepsilon .
$$

The rest of the proof runs as that of $S_{1}$ with a slight modification. We omit here for the similarity. We completes the proof of Theorem 3.1.

\section{Acknowledgements}

We are grateful to the referee for his/her comments and suggestions to this paper. This work was partially supported by the NSF of China (Grant Nos. 11771195, and 12071197), the NSF of Shandong Province (Grant Nos. ZR2019YQ04, 2020KJI002, and 2019KJI003) and the key Laboratory of Complex Systems and Intelligent Computing in University of Shandong (Linyi University).

\section{References}

[1] M. Branmanti and M. Cerutti, $W_{p}^{1,2}$-solvability for the Cauchy-Dirichlet problem for parabolic equations with VMO coefficients, Commun. Partial. Diff. Equ., 18 (1993), 17351763.

[2] F. Beatrous and S. Y. Li, On the boundedness and compactness of operators of Hankel type, J. Funct. Anal., 111 (1993), 350-379.

[3] Y. P. Chen and Y. Ding, A characterization of commutators of parabolic singular integrals, Math. Scand., 108 (2011), 5-25.

[4] Y. P. Chen and Y. Ding, Compactness of the commutators of parabolic singular integrals, Sci. China. Math., 53 (2010), 2633-2648.

[5] Y. P. Chen and Y. Ding, Compactness of commutators of Riesz potential on Morrey spaces, Potential Anal., 30 (2009), 301-313.

[6] Y. P. Chen and Y. Ding, Compactness of commutators for singular integrals on Morrey Spaces, Canad. J. Math., 64 (2012), 257-281.

[7] F. Chiarenza, M. Frasca and P. Longo, $W^{2, p}$-solvability of the Dirichlet problem for nondivergence elliptic equations with VMO coefficients, Trans. Amer. Math. Soc., 336 (1993), 841-853.

[8] Y. Ding and T. Mei, Boundedness and compactness for the commutators of bilinear operators on Morrey spaces, Potential Anal., 42 (2015), 717-748.

[9] Z. W. Fu, Z. G. Liu, S. Z. Lu and H. B. Wang, Characterization for commutators of $n$ dimensional fractional Hardy operators, Sci. China. Math., 50 (2007), 418-426.

[10] Z. W. Fu, S. Z. Lu and S. G. Shi, Two characterizations of central BMO space via the commutators of Hardy operators, Forum Math., 33 (2021), 505-529.

[11] Z. W. Fu, S. Z. Lu and F. Y. Zhao, Commutators of $n$-dimensional rough Hardy operators, Sci. China. Math., 54 (2011), 95-104.

[12] S. Krantz1 and S. Y. Li, Boundedness and compactness of integral operators on spaces of homogeneous type and applications II, J. Math. Anal. Appl., 258 (2001), 642-657.

[13] S. Z. Lu and D. Y. Yan, $L_{p}$-boundedness of multilinear oscillatory singular integrals with Calderon-Zygmund Kernel, Sci. China. Math., 45 (2002), 196-213. 
[14] S. Z. Lu and D. C. Yang, The central BMO spaces and Littlewood-Paley operators, Approx. Theory Appl., 11 (1995), 72-94.

[15] S. G. Shi, Z. W. Fu and S. Z. Lu, On the compactness of commutators of Hardy operators, Pacific J. Math., 307 (2020), 239-256.

[16] S. G. Shi and S. Z. Lu, Characterization of the central Campanato space via the commutator operator of Hardy type, J. Math. Anal. Appl., 429 (2015), 713-732.

[17] E. Stein and G. Weiss, Introdution to Fourier Analysis on Euclidean Spaces, Princeton: Princeton Univ Press, 1971.

[18] Y. Sun, C. Wang and Z. F. Zhang, A Beace-Kato-Majda blow up criterion for the 3-D compressible Navier-Stokes equations, J. Math. Pures Appl., 95 (2011), 36-47.

[19] A. Uchiyama, On the compactness of operators of Hankel type, Tohoku Math. J., 30 (1978), $163-171$.

[20] L. Zhang and S. G. Shi, A characterization of central BMO space via the commutator of fractional Hardy operator, J. Funct. Spaces, 2020 (2020), 1-7. 